\title{
Efeito de diferentes calibres de limas no preparo apical do canal radicular
}

\author{
Elaine Vianna Freitas Fachin*
} Taísa Guimarāes Cassol* *

\begin{abstract}
RESUMO
Estudo in vitro utilizando pré-molares extraídos preparados com calibres crescentes de limas na porçāo apical do canal radicular. Após o preparo, os dentes foram descalcificados, cortados transversalmente em cinco segmentos e avaliados por dois observadores quanto à limpeza, forma e regularidade. Verificou-se que a regiảo apical de dentes de 1 canal tende a ficar mais limpa, circular e regular quando se utilizam limas $n^{\circ} 40$ comparadas com limas menos calibrosas. Em dentes com 2 canais, resultados semelhantes foram alcançados com a lima $n^{\circ} 30$.
\end{abstract}

\section{SUMMARY}

In vitro estudy using extracted bicuspids prepared with increasing file sizes at the apical portion of the root canal. After canal preparation, these teeth were decalcified, serially sectioned and evaluated by two observers regarding cleanliness, shape and regularity. Teeth with one canal tended to be cleaner, more circular and regular as number 40 file is used at the apical

area as compared to smaller size files. Similar findings occured in teeth with two canals when number 30 file is used.

\section{UNITERMOS}

Endodontia, canal radicular, terapia, instrumental odontológico, dente, raiz, estudos de avaliaçāo.

\section{Introdução}

Modernamente inúmeras técnicas de instrumentação têm sido propostas para o preparo mecânico do canal radicular.

A técnica da anticurvatura preconizada por Abou Rass (1) atua na área da curva cervical do canal radicular facilitando a chegada de instrumentos menos calibrosos ao terço apical. O alargamento do terço cervical também é proposto por Goerig (3) com a "step down" technique onde instrumentos calibrosos precedem a técnica "step back" ou escalonada. Walton (9) e também Weine (10) preconizam na técnica escalonada o pré curvamento de limas e a utilização de instrumentos flexíveis de pequeno calibre no terço apical e que progressivamente aumentam de diâmetro em direção cervical. Todas essas técnicas visam o domínio da porção apical do canal radicular que constitui 0 objetivo maior do tratamento endodôntico.
A regularidade da instrumentação apical é altamente buscada. Morgan e Montgomery (7) referem que o preparo circular facilita a obturação, onde todos os nichos dos canais tenham sido trabalhados, já que se deseja uma obturação hermética e sem infiltração apical. Quanto mais limpo, regular e circular estiver o preparo do canal, menor a possibilidade de permanência de restos pulpares ou material necrótico e, consequentemente, melhor será a adaptação de cones de guta percha quando da obturação.

O objetivo do presente estudo é analisar o resultado do trabalho de diferentes calibres de instrumentos (limas tipo Kerr $n^{\circ} 25,30,35$ e 40) no preparo apical do canal radicular comparando cortes transversais de raízes de pré-molares extraídos, em 5 níveis (cervical, médio-cervical, médio, médio-apical e apical) quanto a forma, regularidade e limpeza.

\section{Materiais e Métodos}

Foram selecionados 50 pré-molares superiores extraídos (com 1 e 2 canais), que ficaram mantidos em hipoclorito de sódio a $1 \%$. Os dentes foram autoclavados (MINICLAVE) por 20 minutos a uma temperatura de $121^{\circ} \mathrm{C}$.

Para padronizar os grupos de dentes, as coroas foram cortadas no nível do colo dentário com o auxílio de pontas diamantadas e discos de carborundum.

Os dentes foram divididos em 5 grupos e instrumentados, por um mesmo operador, com limas tipo Kerr até o limite foraminal. A técnica de instrumentação foi a técnica seriada convencional utilizando simultaneamente irrigação com hipoclorito de sódio a $1 \%$ e água oxigenada a $10 \mathrm{vol}$.

\footnotetext{
* Professora Adjunta de Endodontia FO/UFRGS

* Interna em Odontopediatria FO/UFRGS
} 
Os grupos foram classificados da seguinte maneira:

TABELA 1

Distribuiçāo dos dentes em grupos

\begin{tabular}{|c|c|c|c|c|}
\hline GRUPO 0 & controle & s/instr. & 5 dentes ( 1 canal) & 5 dentes ( 2 canais) \\
\hline GRUPO 1 & experim. & instr. ap. =K 25 & 5 dentes ( 1 canal) & 5 dentes ( 2 canais) \\
\hline GRUPO 2 & experim. & instr. ap. $=$ K 30 & 5 dentes ( 1 canal) & 5 dentes ( 2 canais) \\
\hline GRUPO 3 & experim. & instr. ap. =K 35 & 5 dentes ( 1 canal) & 5 dentes (2 canais) \\
\hline GRUPO 4 & experim. & instr. ap. $=\mathrm{K} 40$ & 5 dentes ( 1 canal) & 5 dentes (2 canais) \\
\hline
\end{tabular}

Inicialmente os dentes foram submetidos a descalcificação com Citrato de Sódio a $20 \%$ e Ácido Fórmico a $2 \%$. Para acelerar o procedimento, aumentou-se a concentração do Ácido Fórmico para $45 \%$ e ao final de 28 dias os dentes foram cortados transversalmente com gilete sobre uma laje de vidro em 5 níveis e analisados quanto a morfologia interna (figura 1).

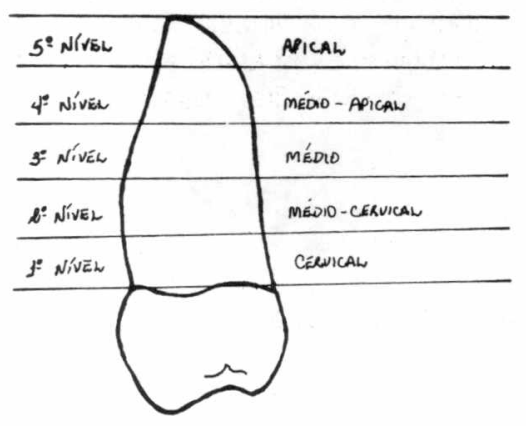

FIGURA 1: Esquema dos segmentos

transversais analisados nos pré-molares

\section{Discussão}

O método da descalcificação utilizado nesse estudo mostrou-se adequado uma vez que permitiu cortes de dentes em cinco níveis iguais (apical, médio-apical, médio, médiocervical, cervical) sem perda de substância dentária tornando a comparação de dentes de um mesmo grupo (pré-molares) mais precisa. mecânico de todos os dentes foi realizado pelo mesmo operador, o que elimina possíveis variáveis decorrentes de diferenças individuais entre técnicos.

A eficiência do solvente orgânico hipoclorito de sódio a $1 \%$ fica evidenciada na medida em que os canais mostraram-se limpos mesmo quando a instrumentação se limitou a limas de pequeno calibre.

A escolha desse grupo de dentes Além disso, o preparo químico-

TABELA 2

Análise de cortes transversais em pré-molares de 1 canal dos grupos controle e experimentais a diferentes níveis

\begin{tabular}{cccccc}
\hline Níveis & $\begin{array}{c}\text { Grupo } \\
\text { Controle }\end{array}$ & Grupo 1 & Grupo 2 & Grupo 3 & Grupo 4 \\
\hline 1 & $\mathrm{~S}, \mathrm{O}, \mathrm{R}$ & $\mathrm{L}, \mathrm{O}, \mathrm{I}$ & $\mathrm{L}, \mathrm{O}, \mathrm{I}$ & $\mathrm{L}, \mathrm{O}, \mathrm{I}$ & $\mathrm{L}, \mathrm{O}, \mathrm{I}$ \\
2 & $\mathrm{~S}, \mathrm{O}, \mathrm{R}$ & $\mathrm{L}, \mathrm{O}, \mathrm{I}$ & $\mathrm{L}, \mathrm{O}, \mathrm{I}$ & $\mathrm{L}, \mathrm{O}, \mathrm{I}$ & $\mathrm{L}, \mathrm{O}, \mathrm{I}$ \\
3 & $\mathrm{~S}, \mathrm{O}, \mathrm{R}$ & $\mathrm{L}, \mathrm{O}, \mathrm{R}$ & $\mathrm{L}, \mathrm{O}, \mathrm{R}$ & $\mathrm{L}, \mathrm{O}, \mathrm{I}$ & $\mathrm{L}, \mathrm{O}, \mathrm{R}$ \\
4 & $\mathrm{~S}, \mathrm{C}, \mathrm{R}$ & $\mathrm{L}, \mathrm{O}, \mathrm{I}$ & $\mathrm{L}, \mathrm{C}, \mathrm{R}$ & $\mathrm{L}, \mathrm{O}, \mathrm{R}$ & $\mathrm{L}, \mathrm{C}, \mathrm{R}$ \\
5 & $\mathrm{~S}, \mathrm{C}, \mathrm{R}$ & $\mathrm{L}, \mathrm{C}, \mathrm{R}$ & $\mathrm{L}, \mathrm{C}, \mathrm{R}$ & $\mathrm{L}, \mathrm{O}, \mathrm{I}$ & $\mathrm{L}, \mathrm{C}, \mathrm{R}$ \\
\hline
\end{tabular}

TABELA 3

Análise de cortes transversais em pré-molares de 2 canais dos grupos controle e experimentais a diferentes níveis

\begin{tabular}{cccccc}
\hline Níveis & $\begin{array}{c}\text { Grupo } \\
\text { Controle }\end{array}$ & Grupo 1 & Grupo 2 & Grupo 3 & Grupo 4 \\
\hline 1 & $\mathrm{~S}, \mathrm{O}, \mathrm{R}$ & $\mathrm{L}, \mathrm{O}, \mathrm{I}$ & $\mathrm{L}, \mathrm{O}, \mathrm{I}$ & $\mathrm{L}, \mathrm{O}, \mathrm{I}$ & $\mathrm{L}, \mathrm{O}, \mathrm{I}$ \\
2 & $\mathrm{~S}, \mathrm{O}, \mathrm{R}$ & $\mathrm{L}, \mathrm{O}, \mathrm{I}$ & $\mathrm{L}, \mathrm{O}, \mathrm{I}$ & $\mathrm{L}, \mathrm{O}, \mathrm{I}$ & $\mathrm{L}, \mathrm{C}, \mathrm{I}$ \\
3 & $\mathrm{~S}, \mathrm{C}, \mathrm{R}$ & $\mathrm{L}, \mathrm{O}, \mathrm{R}$ & $\mathrm{L}, \mathrm{O}, \mathrm{R}$ & $\mathrm{L}, \mathrm{C}, \mathrm{R}$ & $\mathrm{L}, \mathrm{C}, \mathrm{R}$ \\
4 & $\mathrm{~S}, \mathrm{O}, \mathrm{R}$ & $\mathrm{L}, \mathrm{C}, \mathrm{R}$ & $\mathrm{L}, \mathrm{C}, \mathrm{R}$ & $\mathrm{L}, \mathrm{C}, \mathrm{R}$ & $\mathrm{L}, \mathrm{C}, \mathrm{R}$ \\
5 & $\mathrm{~S}, \mathrm{O}, \mathrm{R}$ & $\mathrm{S}, \mathrm{O}, \mathrm{R}$ & $\mathrm{L}, \mathrm{C}, \mathrm{R}$ & $\mathrm{L}, \mathrm{C}, \mathrm{R}$ & $\mathrm{L}, \mathrm{C}, \mathrm{R}$ \\
\hline
\end{tabular}

(pré-molares superiores) deve-se ao fato de possuírem raízes muito achatadas no sentido mésio-distal e, consequentemente, num corte transversal de raiz, apresentarem canais em forma de "gota de lágrima" onde a parte mais estrangulada se situa na porção média do corte. Os achatamentos ocorrem em pré-molares de 1 canal (figura 2) como em pré-molares de 2 canais (figura 3 ).

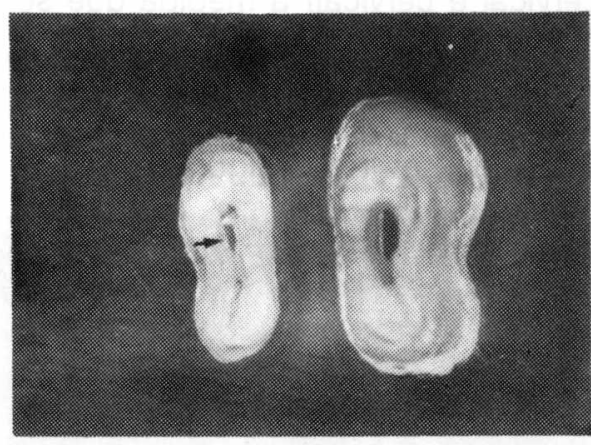

FIGURA 2: Cortes transversais de pré-molar com 1 canal. Detalhe segmentos apical e médio-apical em forma de "gota de lágrima" (seta) (Nikkor Med - Mag. 2x)

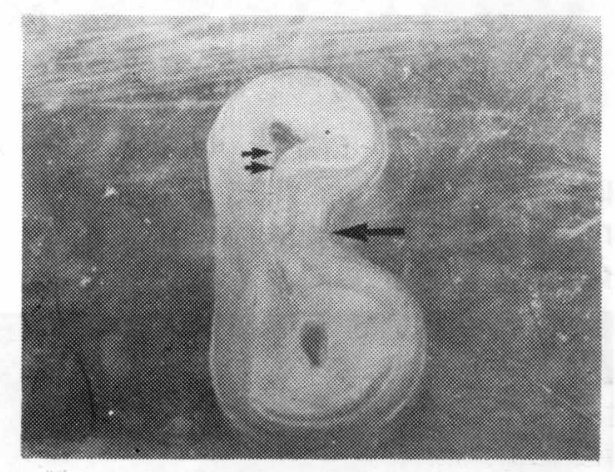

FIGURA 3: Corte transversal de pré-molar com 2 canais. Note achatamento porção

média da raiz (seta) e nicho morfológico do canal radicular (dupla seta)

(Nikkor Med - Mag. 2x)

Essa morfologia abriga resíduos pulpares e constitui um nicho para a proliferação microbiana. Dessa forma o estudo de cortes transversais é apropriado já que permite a análise desses achatamentos, bem como análise da eficiência do preparo químico-mecânico quanto à forma, regularidade e limpeza. Kasahara (6) e colaboradores realizaram análise semelhante utilizando pré-molares superiores com o objetivo de estabelecer critérios de alargamento apical desses dentes.

A presença de irregularidades das paredes do canal, decorre do prepa- 
ro mecânico. $O$ presente estudo revelou irregularidades nas paredes do canal radicular sempre que a instrumentação é realizada. Esses achados também são descritos por Jungmann e Uchin (5) ao referir que nenhuma instrumentação irá com precisāo produzir canais regulares a nível apical. Entretanto, as irregularidades diminuem em todos os niveis (apical, médio-apical, médio, médiocervical e cervical) a medida que se aumenta o calibre da lima. Quanto à forma, no grupo de pré-molares com 1 canal, notou-se que preparos com limas tipo Kerr $n^{\circ} 25$ (figura 4),

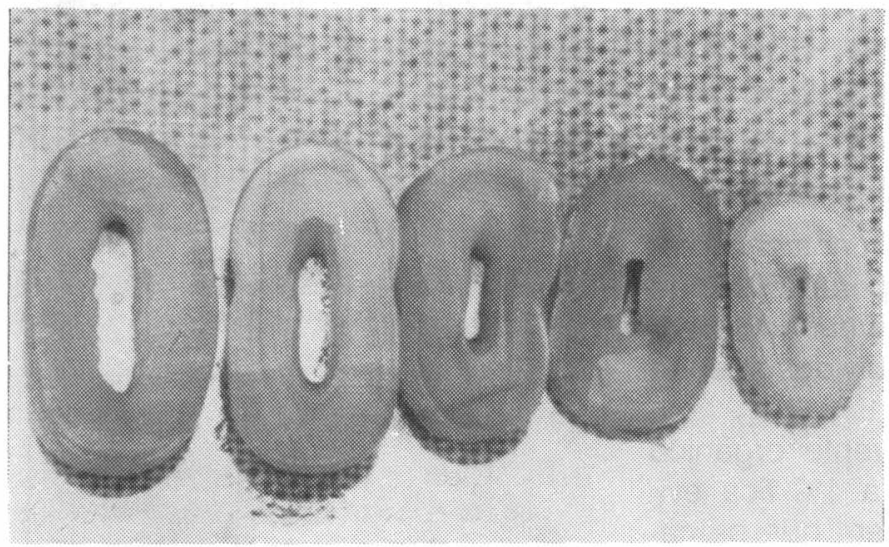

limas $n^{\circ} 30$ e limas $n^{\circ} 35$ (figura 5) no nivel apical, proporcionam canais ovais.

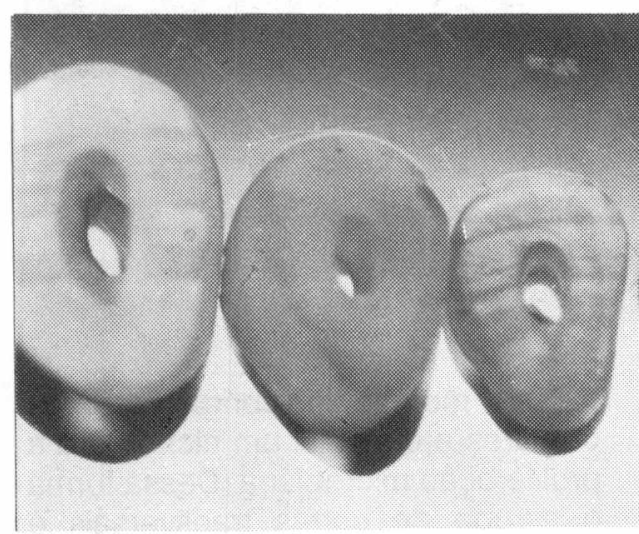

Há uma tendência de se tornarem circulares quando se instrumenta com a lima $n^{\circ} 40$ (figura 6).

Os achados desse estudo demonstram que a lima $n^{\circ} 40$ tende a deixar o canal de pré-molares achatados e com raízes únicas, no nível apical, mais circular, regular e limpo do que as limas de calibres menores (figura 7).

Nesse estudo a lima $n^{\circ} 40$ foi capaz de tocar e arredondar o nicho
FIGURA 6: Cortes transversais de pré-molar com 1 canal, instrumentaçảo seriada lima $n^{\circ}$ 40. (Nikkor Med - Mag. 1.8x)

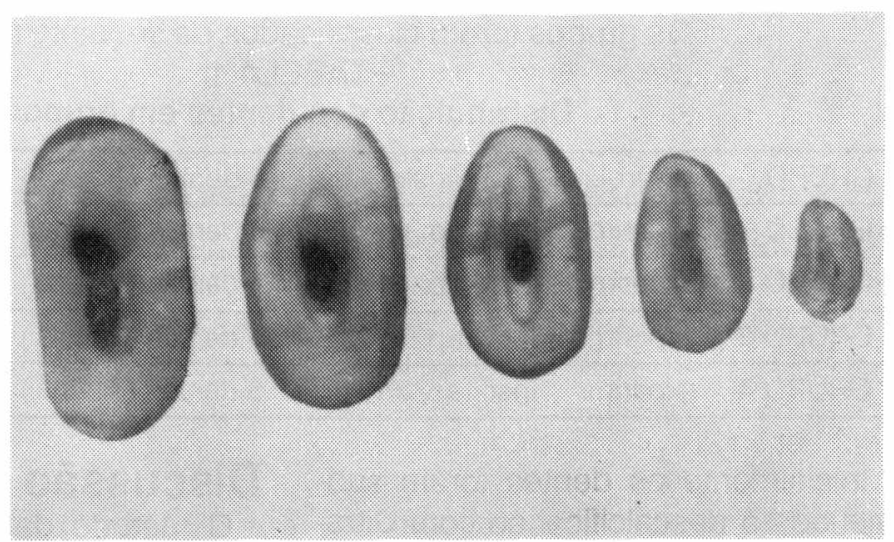

FIGURA 4: Cortes transversais de pré-molar com 1 canal, instrumentação seriada lima $n^{\circ}$ 25. Segmentos apicais ovais, limpos e regulares. (Nikkor Med - Mag. 1/3x)

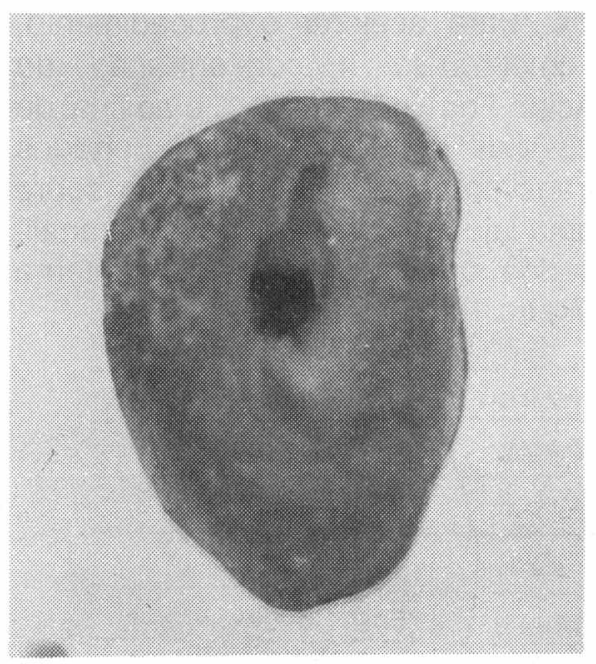

FIGURA 7: Detalhe corte transversal de pré-molar com 1 canal, instrumentação seriada lima $n^{\circ} 40$. Segmento apical canal circular, limpo, regular. (Nikkor Med - Mag. 2x)

finos e curvos não devem ser alargados mais que $\circ \mathrm{n}^{\circ} 25$. Já Ingle e Taintor (4) recomendam uma média de preparo de 40 a 60 como adequado para a maioria dos dentes com canais retos e em canais curvos de 25 a 30.

As irregularidades dos níveis médio, médio-cervical e cervical poderiam ser diminuidas caso se tivesse realizado um preparo escalonado nos níveis supra citados como preconizam Walton (9) e Weine (10) para complementação do preparo seriado onde, então, limas mais calibrosas do que a de $n^{\circ} 40$ tornariam as paredes dos canais mais arredondadas e portanto mais regulares nos cortes transversais. Maior regularidade do terço cervical pode ser obtida com brocas de Batt ou "gates glidden" trabalhando no sentido da anticurvatura do canal no nível cervical. relativamente retos sem chances de perfuraçōes ou degraus. De acordo com Walton (9), canais morfológico presente em raízes achatadas. Achados semelhantes são descritos por Calhoun e Mont(2) onde a lima $n^{\circ} 40$ proc balanceada de Roane (8).

Salienta-se que os achados do presente estudo ocorrem em dentes 
O grupo dos pré-molares com dois canais nos níveis apicais e médioapicais apresentou-se mais limpo, circular e regular a partir da instrumentação com limas tipo Kerr $n^{\circ} 30$. Nesse grupo de dentes a lima $n^{\circ} 30$ já foi capaz de deixar os canais mais circulares. A morfologia interna transversal de dentes com 2 canais apresenta o nicho ou "gota de lágrima" menos pronunciado do que pré-molares de um canal. Dessa forma, nesse estudo, os pré-molares com 2 canais ainda que com raízes achatadas no sentido mésio-distal proporcionaram canais com tendência a circulares e regulares a partir da lima $n^{\circ} 30$ nos níveis apicais e médio-apicais (figura 8). Essa tendência se estende às limas $n^{\circ} 35$ (figura 9) e $n^{\circ} 40$ (figura 10).
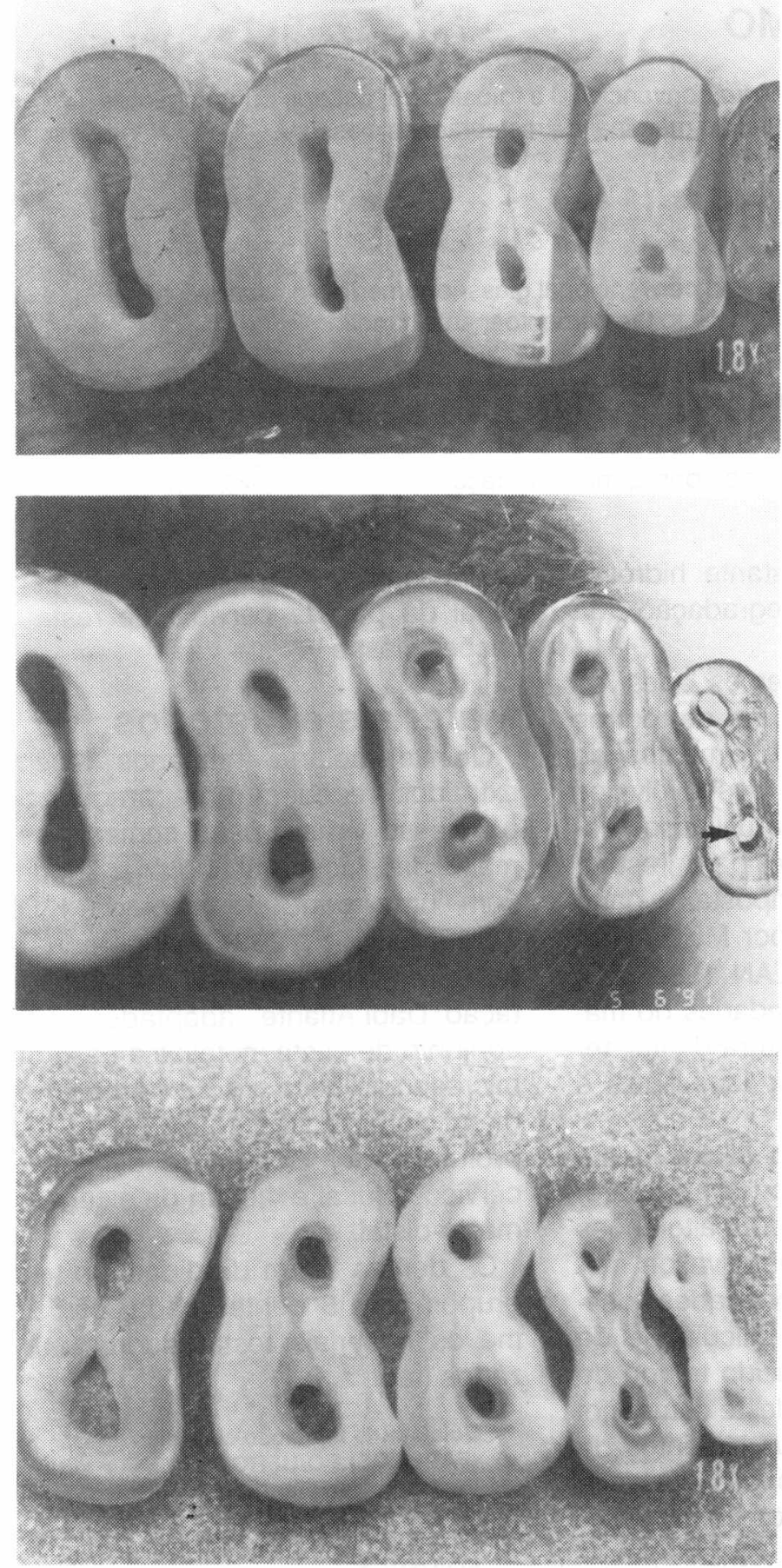

FIGURA 8: Corte transversal de pré-molar com 2 canais, instrumentação seriada lima $n^{\circ}$ 30. Segmento apical médio-apical, canais circulares, limpos e regulares. (Nikkor Med - Mag. 1.8x)

FIGURA 9: Cortes transversais de pré-molar com 2 canais, instrumentaçăo seriada lima $n^{\circ}$ 35. Segmento apical, canais circulares, limpos e regulares (seta). (Nikkor Med - Mag. 2x)

FIGURA 10: Cortes transversais de pré-molar com 2 canais, instrumentação seriada lima $n^{\circ} 40$. Segmentos apicais, canais circulares $\mathrm{e}$ regulares. (Nikkor Med - Mag. $1.8 \mathrm{x})$
Este estudo poderá futuramente ser complementado com a análise de cortes longitudinais. Sugere-se utilizar a mesma metodologia para o estudo do efeito de diferentes calibres de limas, porém com variaçăo de cortes transversais para longitudinais de raiz, comparando resultados e acrescentando dados não só de regularidade, forma e limpeza mas também quanto a possível formação de degraus, "zips" apicais e desvios da posiçăo original do foramen.

\section{Agradecimentos}

As autoras desejam agradecer especialmente o apoio técnico na descalcificação dos dentes à Srta. Isabel da Silva Lauxen e ao Sr. Jorge Reis Furtado pela confecção das fotos.

\section{REFERÊNCIAS BIBLIOGRÁFICAS}

01. ABOU RASS, M.; FRANK, A.; GLICK, D.H The anticurvature filing method to prepare the curved root canal. J. Am. Dent. Assoc., Chicago, v.101, p.792-794 1980.

02. CALHOUN, G.; MONTGOMERY, S. The ef fects of four instrumentation techniques on root canal shape. J. Endodont., Baltimore, v.14, n.6, p.273-277, June, 1988.

03. GOERIG, A.C.; MICHELICH, R.J.; SHULTZ, H.H. Instrumentation of root canals in molar using the step-down technique. J. Endodont., Baltimore, v.8, n.12, p.550-554, Dec., 1982

04. INGLE, J.I.; TAINTOR, J.S. Endodontics. 3.ed. Philadelphia: Lea \& Febiguer, $1985,881 \mathrm{p}$.

05. JUNGMANN, C.L.; UCHIN, R.A.; BUCHER, J.F. Effect of instrumentation on the shape of the root canal. J. Endodont. Baltimore, v.1, n.2, p.66-69, Feb., 1975.

06. KASAHARA, E.; YASUDA, E.; MIYAZANA, A. An evaluation of guidelines for the apical enlargement of maxillary pre-molars. J. Endodont., Baltimore, v.17, n.1, p.1-7, Jan., 1991.

07. MORGAN, L.F.; MONTGOMERY, S. An evaluation of the crown down pressureless technique. J. Endodont., Baltimore, v.10, n.10, p.491-498, Oct., 1984.

08. ROANE, J.B.; SABALA, C.L.; DUCANSON JR, M.G. The balanced force concept for instrumentation of curved canal. J. Endodont., Baltimore, v.11, n.5, p.203-211, May., 1985.

09. WALTON, R.E. Histologic evaluation of different methods of enlargering the pulp canal space. J. Endodont., Baltimore, v.2, n.10, p.304-311, Oct., 1976.

10. WEINE, F.S. et al. Precurved files and incremental instrumentation for root canal enlargement. J. Can. Dent. Assoc. Toronto, v.36, n.4, p.155-157, Apr., 1970. 